\title{
Persistent trigeminal artery as a rare cause of vertebrobasilar insufficiency
}

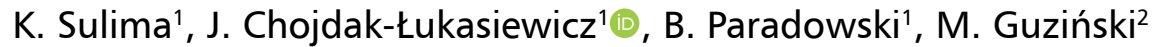 \\ ${ }^{1}$ Department of Neurology, Wroclaw Medical University, Wroclaw, Poland \\ 2Department of General Radiology, Interventional Radiology and Neuroradiology, \\ Wroclaw Medical University, Wroclaw, Poland
}

[Received: 3 April 2021; Accepted: 17 June 2021; Early publication date: 29 June 2021]

\begin{abstract}
The persistent trigeminal artery (PTA) is the most common foetal carotid-basilar anastomosis which may persist into adult life. In the literature there are numerous papers referring to the anatomical characteristics of this anomalous vessel. In the majority of cases PTA is an incidental finding and its clinical significance has been debated. Some authors describe the coincidence of PTA with various clinical syndromes. However, there are few reports linking PTA with the symptoms of vertebrobasilar insufficiency. We present a patient with a 3-year history of recurrent dizziness and impaired vision, precipitated by physical activity, in whom neuroimaging techniques revealed a left PTA. In our case the occurrence of PTA might have been related to the clinical manifestation and the potential mechanism will be discussed. (Folia Morphol 2022; 81, 3: 785-790)
\end{abstract}

Key words: persistent trigeminal artery, computed tomography angiogram, stroke, magnetic resonance imaging, vertebrobasilar insufficiency

\section{INTRODUCTION}

The persistent trigeminal artery (PTA) is the most common remnant of the primitive circulatory system that unites the internal carotid (anterior) and vertebrobasilar (posterior) systems. The PTA contributes to about $85 \%$ of known primitive persistent anastomoses $[1,25]$. The reported incidence of the PTA ranges from $0.1 \%$ to $0.6 \%[7,24]$. Most cases are detected incidentally during brain imaging performed for unrelated reasons, so the real frequency can be higher, based on unrecognised cases. The PTA is usually asymptomatic; however, in some cases may be connected with III or VI nerves palsies, trigeminal neuralgia, vertigo, ataxia, stroke, migraine headache and other neurological symptoms [9, 14, 30, 35]. It may be also associated with other vascular abnormalities such as basilar hypoplasia or anatomical variations of aortic arch and its branches [10, 29].

\section{CASE REPORT}

A 33-year-old man, with no prior comorbidities, presented to our clinic with a 3-year history of recurrent dizziness and impaired vision. The symptoms were transient, lasting usually over a few seconds, triggered by moderate physical activity, especially walking. Head rotation did not aggravate the symptoms. There was no prior history of arm claudication, as well as loss of consciousness or seizures. The patient was an underground copper miner, potentially exposed to dust and toxins. No neurological deficit was found on examination. Magnetic resonance imaging of the brain, with and without contrast enhancement, was

Address for correspondence: J. Chojdak-Łukasiewicz, MD, PhD, Department of Neurology, Wroclaw Medical University, ul. Borowska 213, 50-556 Wrocław, Poland, tel: +48 71734 3100, fax: +48 71734 3109, e-mail: justyna.ch.lukasiewicz@gmail.com

This article is available in open access under Creative Common Attribution-Non-Commercial-No Derivatives 4.0 International (CC BY-NC-ND 4.0) license, allowing to download articles and share them with others as long as they credit the authors and the publisher, but without permission to change them in any way or use them commercially. 


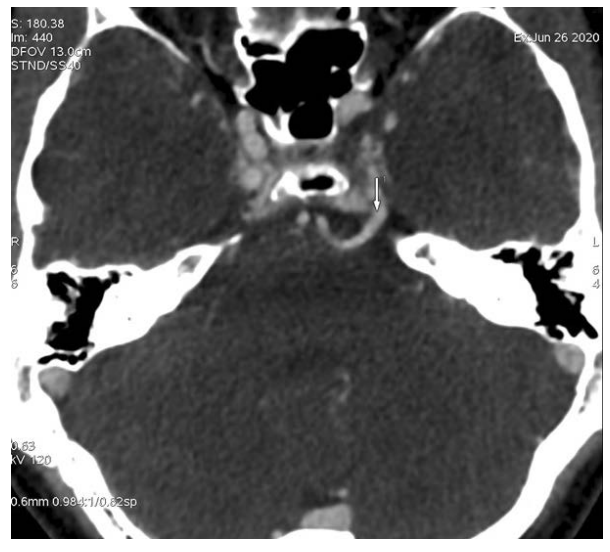

Figure 1. Computed tomography angiogram axial view show persistent trigeminal artery on the left (arrow). It is the most common variant of persistent carotid basilar anastomoses.

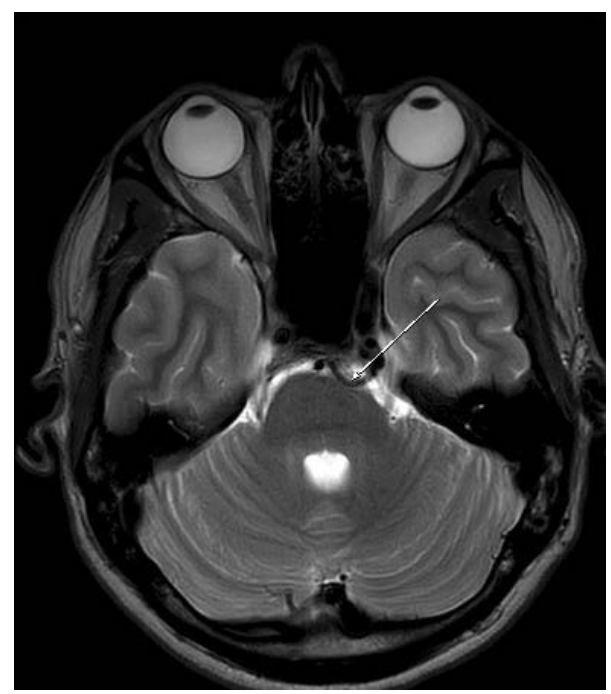

Figure 2. Magnetic resonance. Axial T2-weighted scan. Persistent trigeminal artery on the left (arrow).

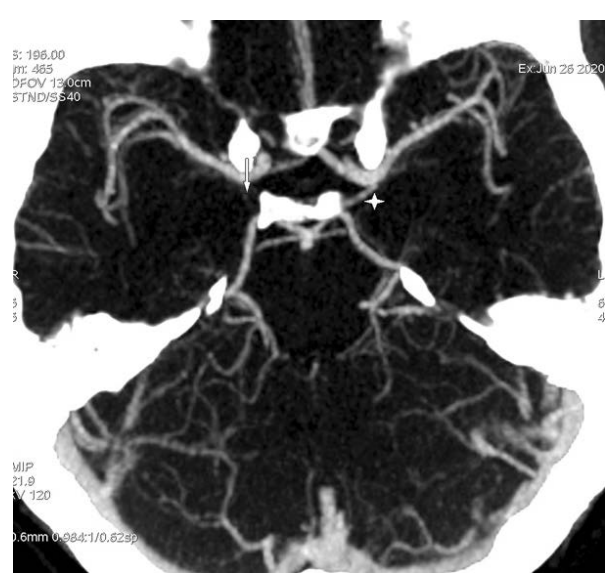

Figure 3. Computed tomography angiogram, maximum intensity projection reconstruction. Persistent trigeminal artery fills superior cerebellar arteries, posterior communicating arteries supplied via patent posterior communicating artery ( $\mathrm{PCoA})$ on the left (asterisk). The absence of right $\mathrm{PC} \mathrm{OA}$ (arrow).

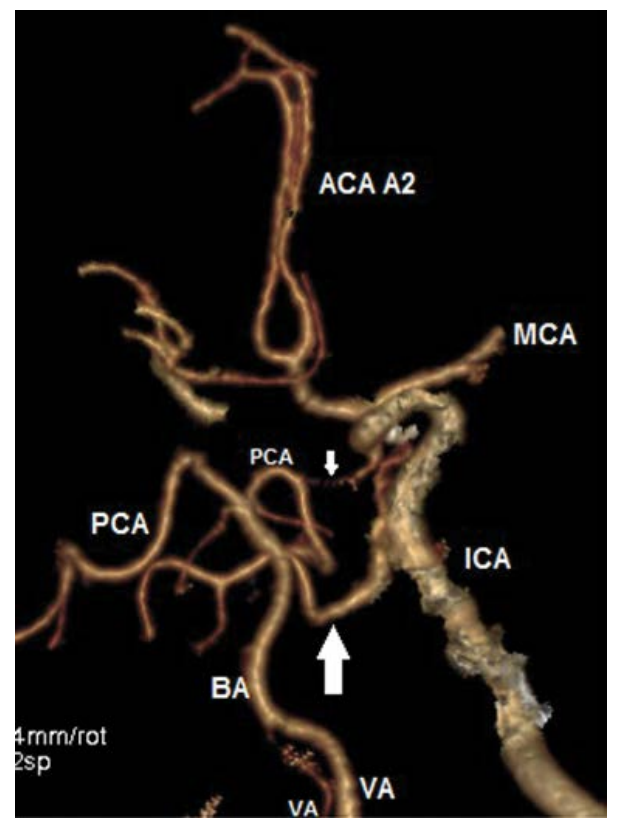

Figure 4. Volume rendering three-dimensional reconstruction, cerebral angiogram based on computed tomography angiogram examination. Bigger arrow persistent trigeminal artery (connection basilar artery [BA]-internal carotid artery [ICA]), smaller arrow posterior communicating artery (connection posterior communicating artery [PCA]-internal carotid artery [ICA]), both on left side; ACA - anterior cerebral artery; MCA — middle cerebral artery; VA - vertebral artery.

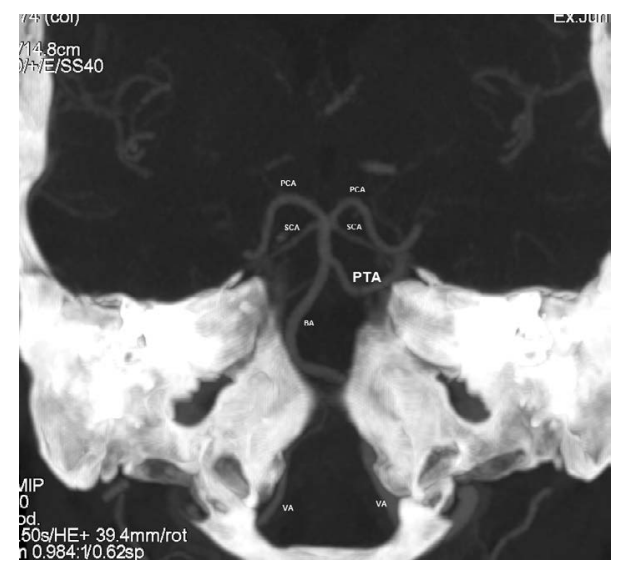

Figure 5. Posterior circulation computed tomography angiogram, maximum intensity projection oblique reconstruction; $\mathrm{BA}$ - basilar artery; PCA — posterior communicating artery; PTA — persistent trigeminal artery; SCA — superior cerebellar artery.

within the normal limits. A computed tomography angiogram was performed, revealing a left PTA, an anomalous vessel extending from the internal carotid artery (ICA) to the basilar artery (BA) (Fig. 1-5). The PTA arose from the junction between petrous and cavernous ICA, and ran posterolaterally along the trigeminal 


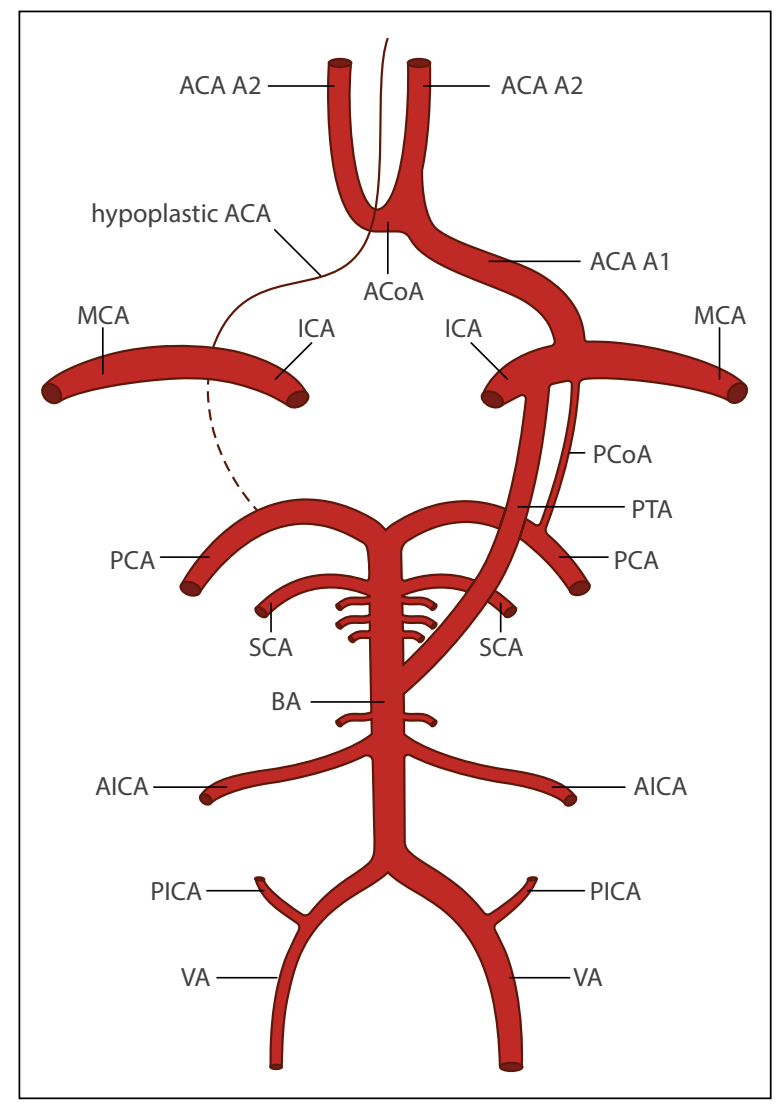

Figure 6. Schematic presentation of the cerebral vasculature anatomy in our patient; ACA A1 - A1 segment of anterior cerebral artery; ACA A2 - A2 segment of anterior cerebral artery; $A C o A-$ anterior communicating artery; $\mathrm{PCo}$ - posterior communicating artery; ICA — internal carotid artery; AICA — anterior inferior cerebellar artery; PICA — posterior inferior cerebellar artery; BA — basilar artery; MCA — middle cerebral artery; PCA — posterior communicating artery; PTA — persistent trigeminal artery; SCA — superior cerebellar artery; VA — vertebral artery

nerve, its diameter was about 2-3 mm. The PTA filled superior cerebellar arteries (SCAs), while the patent left posterior communicating artery (PCoA) supplied the posterior cerebral arteries (PCAs). According to its course and connections, the vessel was classified as Saltzman type II. The examination also showed the focal stenosis of the right vertebral artery (VA) at the C4 level, the absence of the right PCoA and hypoplasia of A1 segment of anterior cerebral artery (ACA) (Fig. 2). The Figure 6 is a pictorial presentation of the cerebral vasculature anatomy in our patient. No other variations were found in the vessels supplying the central nervous system. The patient was treated conservatively with acetylsalicylic acid ( $75 \mathrm{mg}$ per day) and cinnarizine with dimenhydrinate $(20+40 \mathrm{mg}$ 3 times per day) as a short-term therapy for dizziness.
The potential benefits of surgical procedure would not justify the possible risk of such treatment.

\section{DISCUSSION}

There are many reports regarding intracranial vascular development, and one of the most significant was made by Padget in 1948 [2, 32]. During the evolution of the vertebrobasilar arterial system, the hindbrain is supplied predominantly by four foetal carotid-basilar anastomoses: the trigeminal, otic, hypoglossal, and proatlantal intersegmental arteries, arising at the $3 \mathrm{~mm}$ embryonic stage. The above-mentioned connections regress with the subsequent development of posterior communicating arteries and basilar artery and should disappear by the $14 \mathrm{~mm}$ stage [2]. Failure of this process results in persistence of these vessels into adult life. Within four persistent foetal carotid-basilar anastomoses, the trigeminal artery is the most common.

The PTA was firstly reported by Richard Quinn in 1844 during an autopsy [35]. In 1950 the PTA was recognised by Sutton, based on angiography [35].

The PTA typically arises from the posterior or lateral part of intracavernous (C4) segment of the ICA and terminates at the BA, between the first part of the SCA and anterior inferior cerebellar artery (AICA) [4, 12, 34]. In some cases pontine perforating branches of PTA are observed $[3,23,33]$. The incidence of PTA varies from $0.1 \%$ to $0.6 \%$ [11] and according to some studies is higher in women [5, 22]. In most cases the right or none-sided predominance of the PTA is detected.

The most common classification system, based on angiographic characteristics, was introduced by Georg-Frederik Saltzman in 1959 [4]. It divides the PTA into three groups according to its relationship to the basilar artery. In Salzmann type I, the PTA enters the basilar artery between the SCA and the AICA, supplying both PCA and SCA. The BA is typically hypoplastic and the ipsilateral posterior communicating artery is absent. In Saltzman type II, the PTA provides the anterior superior cerebellar artery, whereas the posterior cerebral arteries arise from the posterior communicating arteries. The first segment of BA is missing. Saltzman type III, the rarest type, is considered to be a combination of types I and II. In type III PCA is supplied by the ipsilateral PCoA, the other PCA and both SCA being supplied by the PTA. Saltzman type $I$ is the most prevalent and accounts for about $24 \%$ of all cases $[29,35]$. 
In 1998 Salas et al. [33] proposed a different classification, dividing the PTA into two types based on its relationship to the abducens nerve: the lateral (petrosal) and medial (sphenoidal). The lateral type is recognised when the artery courses laterally to the abducens nerve and pierces the dura mater just medially to the sensory root of the trigeminal nerve. The second, medial type, is recognised when the artery courses medially to the abducens nerve, penetrates the dorsum sellae and joins the distal third of the basilar artery [33]. The study indicated that the latter type is more common [31, 36].

Most cases of PTA are recognised incidentally in imaging diagnostic tests performed for other reasons. Typically PTA is associated with BA hypoplasia, the observation being confirmed in the large neuroimaging series $[10,31]$. There are some reports in the literature regarding the correlation of the PTA with other abnormalities in the circle of Willis, such as the absence of the ipsilateral PCoA, VA or BA. Due to embryonic error in vasculogenesis, the PTA is frequently observed in PHACE syndrome [19]. The connection with the increased risk of aneurysm is controversial. The high incidence of aneurysm is explained by haemodynamic changes in blood flow, which promote aneurysm formation [31]. In an older study [1, 18], the prevalence of associated intracranial aneurysms in patients with PTA was reported to be up to $14-32 \%$. In the newest reports based on magnetic resonance imaging examination, the prevalence is estimated to be approximately $16 \%$ [12]. However, Meckel et al. [29] suggested that the real frequency of aneurysm coexisting with the PTA does not differ from that in the general population ( $4.2 \%$ vs. $3.7 \%)[25,29]$. Aneurysm connected with the PTA has a lot of potential sites. Behari described an aneurysm on the posterior communicating artery, Maeshima et al. [28] showed a case of PTA connected with multiple aneurysms on ACA and middle cerebral artery $[8,28]$. Bechri et al. [7] in 2020 reported a case of a posterior meningeal artery aneurysm coexisting with PTA. In a great number of cases the persistent trigeminal artery is an incidental discovery and its clinical significance has been debated. The symptoms may depend on associated vascular abnormalities or their localisation. The PTA has been identified as a rare cause of cranial nerves dysfunction, including incomplete oculomotor and abducens nerve palsies, trigeminal neuralgia and hemifacial spasm. The ischaemic events related to the PTA are rare and can occur through the steal phe- nomenon or thrombosis in anterior circulation [16, 21]. Typically, persistent trigeminal artery aneurysms (PTAA) are discovered incidentally in patients with subarachnoid haemorrhage (SAH) due to the rupture of a simultaneous aneurysm in another localisation. Ruptured PTAA can be presented with symptoms of headache and posterior cranial fossa symptoms, according to the SAH [29]. In the case of large and giant cavernous PTAAs, cranial neuropathy or facial pain can be observed [13]. Furthermore, PTA was postulated as a potential cause of vertebrobasilar insufficiency (VBI), which could be defined as inadequate blood flow through the posterior circulation system, without actual infarction, with a temporary inability to meet cerebral metabolic needs as a consequence [20]. Posterior circulation supplies the brainstem, cerebellum, thalamus, occipital lobe, some portions of temporooccipital and parietooccipital junctions and even the inner ear through the labyrinthine artery [6]. Dizziness is the most common symptom of VBI, but the diversity of brain regions being supplied by vertebrobasilar vasculature explains the variety of complaints, such as diplopia, blindness, visual field deficits, imbalance, and ataxia [20]. Patients with VBI usually present more than one symptom at the same time. The most frequent pathology underlying VBI is atherosclerosis; however, other causes such as embolism, arterial dissection, migraine and fibromuscular dysplasia may occur [27]. As during embryonic development PTA originates from ICA, the physiological direction of blood flow through PTA is mostly from the ICA to the BA $[6,15]$. Nevertheless, the reverse of this direction may occur in certain clinical situations. In case of severe stenosis or occlusion of the ipsilateral ICA, the reversal of flow through PTA may play a protective role and be sufficient to perfuse ICA and its branches, keeping the patient free of hypoperfusion symptoms [26]. Conversely, if PTA coexists with other anomalies of the intracranial vessels, such as hypoplastic vertebral artery, ICA may be the sole supply to the posterior circulation system. In that case if carotid stenosis occurs, the patient may develop symptoms of VBI [6]. However, our patient did not have any risk factors for cardiovascular disease, and there were also no radiologic signs of atherosclerosis. The review of the literature revealed only one previously reported case linking PTA with VBI symptoms, without the simultaneous presence of carotid pathology, in which dilatation of PTA and BA was the speculated mechanism [17]. In our patient 
the lack of the right PCoA, together with the stenosis of the right VA, might have resulted in the left ICA being the main supply to the posterior circulation system. In the case of the exercise-induced increase of metabolism in the anterior portion of the brain, PTA might have been insufficient to perfuse the posterior fossa region of the brain, leading to VBI symptoms. We could not exclude the potential reverse of blood flow direction in this case, from the vertebrobasilar system to the carotid artery.

\section{CONCLUSIONS}

Due to the high prevalence of dizziness in the general population, awareness of its rare causes seems to be crucial. The PTA may play a role in the production of VBI symptoms. In patients with VBI of unknown origin, a computed tomography angiogram should be considered as a diagnostic procedure.

\section{Conflict of interest: None declared}

\section{REFERENCES}

1. Agnoli AL. Vascular anomalies and subarachnoid haemorrhage associated with persisting embryonic vessels. Acta Neurochir (Wien). 1982; 60(3-4): 183-199, doi: 10.1007/ BF01406306, indexed in Pubmed: 7072535.

2. Ali S, Radaideh MM, Shaibani A, et al. Persistent trigeminal artery terminating in the posterior inferior cerebellar artery: case report. Neurosurgery. 2008; 62(3): E746-8; discussion E746, doi: 10.1227/01.neu.0000317327.17225. f8, indexed in Pubmed: 18425001.

3. Alcalá-Cerra G, Tubbs RS, Niño-Hernández LM. Anatomical features and clinical relevance of a persistent trigeminal artery. Surg Neurol Int. 2012; 3: 111, doi: 10.4103/21527806.101798, indexed in Pubmed: 23087827.

4. Arráez-Aybar LA, Fuentes-Redondo T, Millán JM. Persistent trigeminal artery: a cross-sectional study based on over 3 years conventional angiography, $\mathrm{CT}$ angiography and MR angiography images. Surg Radiol Anat. 2016; 38(4): 445-453, doi: 10.1007/s00276-015-1578-5, indexed in Pubmed: 26499125.

5. Bai M, Guo Q, Li S. Persistent trigeminal artery/persistent trigeminal artery variant and coexisting variants of the head and neck vessels diagnosed using 3 T MRA. Clin Radiol. 2013; 68(11): e578-e585, doi: 10.1016/j. crad.2013.05.099, indexed in Pubmed: 23845929.

6. Battista RA, Kwartler JA, Martinez DM. Persistent trigeminal artery as a cause of dizziness. Ear Nose Throat J. 1997; 76(1): 43-45, indexed in Pubmed: 9018936.

7. Bechri H, Louraoui SM, Fikri M, et al. Persistence of a trigeminal artery associated with a posterior meningeal artery aneurysm: case report and literature review. J Surg Case Rep. 2020; 2020(2): rjz389, doi: 10.1093/jscr/rjz389, indexed in Pubmed: 32047593.

8. Behari S, Krishna H, Kumar MV, et al. Association between an aplastic basilar artery, unaccompanied by a primitive carotid-vertebrobasilar anastomosis, and multiple aneurysms on the dominant posterior communicating artery. J Neurosurg. 2004; 100(5): 946-949, doi: 10.3171/jns.2004.100.5.0946, indexed in Pubmed: 15137614.

9. Bosco D, Consoli D, Lanza PL, et al. Complete oculomotor palsy caused by persistent trigeminal artery. Neurol Sci. 2010; 31(5): 657-659, doi: 10.1007/s10072-010-0342-1, indexed in Pubmed: 20552240.

10. Boyko OB, Curnes JT, Blatter DD, et al. MRI of basilar artery hypoplasia associated with persistent primitive trigeminal artery. Neuroradiology. 1996; 38(1): 11-14, doi: 10.1007/ BF00593207, indexed in Pubmed: 8773267.

11. Brzegowy K, Pękala PA, Zarzecki MP, et al. Prevalence and clinical implications of the primitive trigeminal artery and its variants: a meta-analysis. World Neurosurg. 2020; 133 : e401-e411, doi: 10.1016/j.wneu.2019.09.042, indexed in Pubmed: 31536812.

12. Chen YC, Li MH, Chen SW, et al. Incidental findings of persistent primitive trigeminal artery on 3-dimensional time-of-flight magnetic resonance angiography at 3.0 T: an analysis of 25 cases. J Neuroimaging. 2011; 21(2): 152-158, doi: 10.1111/j.1552-6569.2010.00472.x, indexed in Pubmed: 20331497.

13. Cloft HJ, Razack N, Kallmes DF. Prevalence of cerebral aneurysms in patients with persistent primitive trigeminal artery. J Neurosurg. 1999; 90(5): 865-867, doi: 10.3171/ jns.1999.90.5.0865, indexed in Pubmed: 10223452.

14. Engelhardt J, El Hage G, Bojanowski MW. Persistent trigeminal artery as collateral circulation in ischemic stroke. World Neurosurg. 2021; 148: 67-69, doi: 10.1016/j. wneu.2021.01.034, indexed in Pubmed: 33476776.

15. Enomoto T, Sato A, Maki Y. Carotid-cavernous sinus fistula caused by rupture of a primitive trigeminal artery aneurysm. Case report. J Neurosurg. 1977; 46(3): 373-376, doi: 10.3171/jns.1977.46.3.0373, indexed in Pubmed: 839263.

16. Ferreira A, Coelho PS, Cruz VT. Persistent trigeminal artery in a patient with posterior circulation stroke treated with rt-PA: case report. BMC Neurol. 2019; 19(1): 257, doi: 10.1186/s12883-019-1492-2, indexed in Pubmed: 31656167.

17. Fields WS. The significance of persistent trigeminal artery. Carotid-Basilar anastomosis. Radiology. 1968; 91(6): 1095-1101, indexed in Pubmed: 5699609.

18. George AE, Lin JP, Morantz RA. Intracranial aneurysm on a persistent primitive trigeminal artery. Case report. J Neurosurg. 1971; 35(5): 601-604, doi: 10.3171/ jns.1971.35.5.0601, indexed in Pubmed: 5120008.

19. Heyer GL, Dowling MM, Licht DJ, et al. The cerebral vasculopathy of PHACES syndrome. Stroke. 2008; 39(2): 308-316, doi: 10.1161/STROKEAHA.107.485185, indexed in Pubmed: 18174492.

20. Hirschberg $M$, Hofferberth B. Calcium antagonists in an animal model of vertebrobasilar insufficiency. Acta Otolaryngol Suppl. 1988; 460: 61-65, doi: 10.3109/00016488809125136, indexed in Pubmed: 3074620.

21. lancu $D$, Anxionnat $R$, Bracard $S$. Brainstem infarction in a patient with internal carotid dissection and persistent trigeminal artery: a case report. BMC Med Imaging. 2010; 10: 14, doi: 10.1186/1471-2342-10-14, indexed in Pubmed: 20598138. 
22. Kim MJ, Kim MS. Persistent primitive trigeminal artery: analysis of anatomical characteristics and clinical significances. Surg Radiol Anat. 2015; 37(1): 69-74, doi: 10.1007/s00276014-1318-2, indexed in Pubmed: 24899147.

23. Khodadad G. Trigeminal artery and occlusive cerebrovascular disease. Stroke. 1977; 8(2): 177-181, doi: 10.1161/01. str.8.2.177, indexed in Pubmed: 847781.

24. Komiyama M. Persistent trigeminal artery and its variants. Interv Neuroradiol. 2019; 25(6): 635-637, doi: 10.1177/1591019919863110, indexed in Pubmed: 31296065.

25. Lam JJ, Shah MT, Chung SLi, et al. Persistent primitive trigeminal artery associated with a cavernous carotid aneurysm. Case report and literature review. J Radiol Case Rep. 2018; 12(11): 1-11, doi: 10.3941/jrcr.v12i11.3500, indexed in Pubmed: 30647831.

26. Lewis VL, Cail WS. Persistent trigeminal artery with internal carotid artery occlusion. Neurosurgery. 1983; 13(3): 314-315, doi: 10.1227/00006123-198309000-00018, indexed in Pubmed: 6621844.

27. Lima Neto AC, Bittar R, Gattas GS, et al. Pathophysiology and diagnosis of vertebrobasilar insufficiency: a review of the literature. Int Arch Otorhinolaryngol. 2017; 21(3): 302-307, doi: 10.1055/s-0036-1593448, indexed in Pubmed: 28680502.

28. Maeshima S, Tereda T, Masuo O, et al. Multiple cerebral aneurysms with persistent primitive trigeminal artery. J Clin Neurosci. 1999; 6(1): 52-54, doi: 10.1016/s09675868(99)90606-9.

29. Meckel S, Spittau B, McAuliffe W. The persistent trigeminal artery: development, imaging anatomy, variants, and associated vascular pathologies. Neuroradiology. 2013;
55(1): 5-16, doi: 10.1007/s00234-011-0995-3, indexed in Pubmed: 22170080.

30. Momma F, Ohara S, Ohyama T. Persistent trigeminal artery associated with brainstem infarct--case report. Neurol Med Chir (Tokyo). 1992; 32(5): 289-291, doi: 10.2176/ nmc.32.289, indexed in Pubmed: 1378946.

31. O'uchi E, O'uchi T. Persistent primitive trigeminal arteries (PTA) and its variant (PTAV): analysis of 103 cases detected in 16,415 cases of MRA over 3 years. Neuroradiology. 2010; 52(12): 1111-1119, doi: 10.1007/s00234-010-0669-6, indexed in Pubmed: 20309534.

32. Padget DH. The development of the cranial arteries in the human embryo. Contribution to embryology. Carnegie Institution. 1948; 205-261.

33. Salas E, Ziyal IM, Sekhar LN, et al. Persistent trigeminal artery: an anatomic study. Neurosurgery. 1998; 43(3): 557-561; discussion 561, doi: 10.1097/00006123-19980900000082, indexed in Pubmed: 9733310.

34. Tubbs RS, Verma K, Riech S, et al. Persistent fetal intracranial arteries: a comprehensive review of anatomical and clinical significance. J Neurosurg. 2011; 114(4): 1127-1134, doi: 10.3171/2010.11.JNS101527, indexed in Pubmed: 21235309.

35. Tyagi G, Sadashiva N, Konar S, et al. Persistent Trigeminal Artery: Neuroanatomic and Clinical Relevance. World Neurosurg. 2020; 134: e214-e223, doi: 10.1016/j. wneu.2019.10.025, indexed in Pubmed: 31627002.

36. Uchino A, Saito N, Okada $Y$, et al. Persistent trigeminal artery and its variants on MR angiography. Surg Radiol Anat. 2012; 34(3): 271-276, doi: 10.1007/s00276-0110848-0, indexed in Pubmed: 21739246. 